\title{
Acoustic Characterisation of Towing Tanks
}

\author{
Henry Way ${ }^{*}$, Phillip Joseph ${ }^{2}$, Stephen Turnock ${ }^{1}$, Ronnie Leung ${ }^{3}$, Victor Humphrey ${ }^{2}$ \\ ${ }^{1}$ Fluid Structure Interactions Research Group, University of Southampton, Southampton, United Kingdom \\ ${ }^{2}$ Institute of Sound and Vibration Research, University of Southampton, Southampton, United Kingdom \\ ${ }^{3}$ Dstl, Fareham, United Kingdom
}

Large water tanks known as towing tanks, wave basins and cavitation tunnels, are used to determine the performance of ships, boats and submarines at model-scale. They may also be used for noise measurements but suffer from the effects of reverberation, which makes it difficult to capture the free field conditions necessary for complete characterisation of the noise. This paper describes an adapted Image Source Model and its use to understand these reverberation effects. It extends the conventional Image Source Model by the use of spherical wave reflection coefficients at the tank boundaries, in addition to the inclusion of volumetric absorption. Validation of the Image Source Model is undertaken by comparison with published transfer function measurements made in the depressurized wave basin at the Maritime Research Institute Netherlands (MARIN). The relative contribution to the tank response from the direct field and free surface is examined. The tank response is found to vary with three frequency-scales (periodicities) resulting from interactions with the free surface and axial modes.

\section{Introduction}

Towing tanks are commonly used to measure the hydrodynamic performance of ships at model scale. Acoustic performance can also be measured by introducing hydrophones to measure acoustic radiation from the model being towed. However, the tank, which is typically more than $100 \mathrm{~m}$ in length, and is constructed from concrete, can significantly modify the acoustic field compared with the radiation that would occur in a free field environment in the absence of reflections.

The acoustic behaviour of a towing tank lies somewhere between a long duct and a room. Their acoustic response is complicated by the presence of acoustic modes across the duct cross-section and along the length of the towing tank, the presence of head waves for waves incident at low grazing angles to the concrete walls, and high levels of destructive interference at the water - air upper surface (Lloyd's mirror effect).

This paper describes an analytic model to predict the acoustic response of towing tanks. The model is a combination of a classical image model with the use of a plane-wave decomposition for the images closes to the receiver, where spherical wave effects are most significant. This model will allow the influence of the tank on acoustic measurements to be predicted and hence aid the development of techniques for removing the effects of reverberation on acoustic measurements in towing tanks.

Acoustic measurements made in water tanks suffer from the effects of reverberation. Many measurement facilities exist to make these measurements, which are primarily large water tanks known as towing tanks, wave basins or cavitation tunnels. Reverberation modifies the pressure level measured by a simple hydrophone and results in a highly biased estimate of the acoustic source strength. To correct for the effect of reverberation in the measurement facility, and obtain an equivalent free field result, a detailed 
understanding of the underwater acoustic properties of the facility used for experimentation is needed. Previous literature has corrected for reverberation by taking measurements within the reverberation radius of the source, where the direct field is dominant over the reverberant field. This inevitably requires the measurements to be made in the source near field, which introduces its own sources of error. The effect of the pressure release free surface is also corrected for by removing the interference pattern of a simple point source under a free surface, from the measured pressure levels [1][2].

This paper presents a numerical method for predicting the acoustic response of water tanks, with special emphasis on long towing tanks. The basis of the model is a modification of the classical Image Source Model, performed in the frequency domain. This paper demonstrates that the contribution to the sound field from image sources well away from the measurement point is adequately represented by the plane wave reflection at the appropriate angle. However, image sources relatively close to the receiver, whose contribution to the sound field is greatest, require a plane wave decomposition of the spherical wave field over all angles to include the effects of wavefront curvature and its interaction with the plane boundaries.

This paper begins with a general discussion of the acoustical physics of a water tank. Section 2 is an outline of the Image Source Model, which is able to capture the essential behaviour of the sound field in the water tank. Section 3 is a validation of the Image Source Model, through comparison between the analytical solutions and published measurements from [2]. Section 4 compares the simulated transfer functions between reference and receiver hydrophones for 4 different sized towing tanks. Section 5 provides a brief overview of the use of the Image Source Model to obtain free field conditions from measurements inside a reverberant underwater environment. Sections 6 and 7 provide an outline of future work and conclusions.

\subsection{The Acoustic Environment of a Water Tank}

A water tank consists of a volume of fluid enclosed by five solid walls and a free upper surface representing the water - air interface. When an acoustic disturbance radiates acoustic energy continuously into the tank, an energy balance occurs. All of the acoustical energy radiated by the source is either transmitted into the tank walls or is transferred into heat energy through volumetric absorption of the water.

The impedance difference between the fluid and the solid walls leads to a partially reflected acoustic wave, with some of the wave's energy transmitted into the wall. The specular acoustic reflection coefficient of a fluid-solid interface has been outlined by Brekhovskikh [3], in which the effects of longitudinal and shear (transverse) waves in the solid are taken into account. At the free surface a pressure release boundary condition applies and the reflected wave undergoes total internal reflection with a phase change of 180 degrees. The interference pattern due to the interaction between the direct field of a source and the reflection from the free surface is known as the Lloyd's mirror effect and leads to strong interference effects.

\subsection{Numerical Methods}

A number of numerical methods are available for predicting the acoustic pressure field of a room due to an acoustic source. The Image Source Method is a conceptually simple method, which can be adapted to include irregular geometry, energy loss to the walls and in the fluid through volumetric absorption. Allen 
and Berkley [4] showed that the Green's function for a rectangular room with rigid walls is equivalent to a time or frequency domain solution based on the image method.

The Image Source Model assumes specular reflection at the walls and therefore cannot capture diffuse reflections. For a rectangular room, the Image Source Model is a simple analysis tool. However, for room shapes that are not perfectly rectangular, some of the images can have an invalid ray-path, i.e. the ray would not physically reach the measurement location. Ray-tracing techniques can be used to find invalid paths and remove them. Towing tanks have very regular geometry and can be closely approximated as a perfectly rectangular room, hence in this paper, there are no invalid image paths to be removed.

Reflection coefficients can be used in the Image Source Model to simulate rooms with non-acoustically rigid walls. Volumetric absorption can be included in the Image Source Model, through the addition of a frequency dependent absorption coefficient in the exponential term of the monopole equation. The Image Source Model described in this paper, will include angle and frequency dependent reflection coefficients, as well as including further energy loss through volumetric absorption.

Normal-mode theory is another numerical method which may be useful for simulating a water tank's acoustical behaviour. This method assumes rigid walls and a frequency-dependent damping term. However, as will be shown in Section 2.1, the concrete walls of a towing tank do not provide a hard wall boundary condition. Normal-mode theory can give an approximation for the modal frequencies of the tank, $f_{l, m, n}$, assuming hard walls and a free surface, given by

$$
f_{l, m, n}=\frac{c}{2} \sqrt{\left[\frac{l}{L_{x}}\right]^{2}+\left[\frac{m}{L_{y}}\right]^{2}+\left[\frac{(2 n+1)}{2 L_{z}}\right]^{2}}
$$

where $L_{x}, L_{y}, L_{z}$ are the tanks dimensions and $l, m, n$ are real integers between 0 and $\infty$ and $c$ is the speed of sound in $m s^{-1}$. This paper will show that although a towing tank does not have a hard wall boundary condition, there exist modes at frequencies close to those given by the normal-mode theory approximation.

\section{Adapted Image Source Model}

The adapted Image Source Model, is able to predict the acoustic frequency response due to a point monopole source, for an arbitrary source and receiver location inside a towing tank. Dipole and multipole fields can be modelled by the principle of linear superposition of monopoles. Propeller noise, can typically be represented by monopole and dipole sources for cavitation noise and the noise due to fluctuating forces over the propeller blade, respectively. The model in this paper is therefore able to calculate a towing tank's response to propeller cavitation noise source, under the following source assumptions:

1. The source is stationary (not rotating or translating);

2. The walls of the tank are smooth relative to the wavelength emitted, such that reflections are specular and not diffuse;

3. There is no scattering or diffraction from other obstacles in the tank;

4. The tank's geometry is rectangular; 
The adapted Image Source Model is also useful for calculating the transfer the function between a reference and receiver hydrophone, so that the source's response in free-field conditions can be predicted.

Consider a rectangular water tank of dimensions $L_{x}, L_{y}$ and $L_{z}$, with the origin located in the bottom left of the tank, as seen in Figure 1. The tank has an open free surface on the plane $z=L_{z}$, and hard walls on the planes: $x=0, L_{x} y=0, L_{y}$ and $z=0$. It is assumed that each wall is infinitely thick, such that sound does not re-enter the tank via reflections from secondary interfaces.

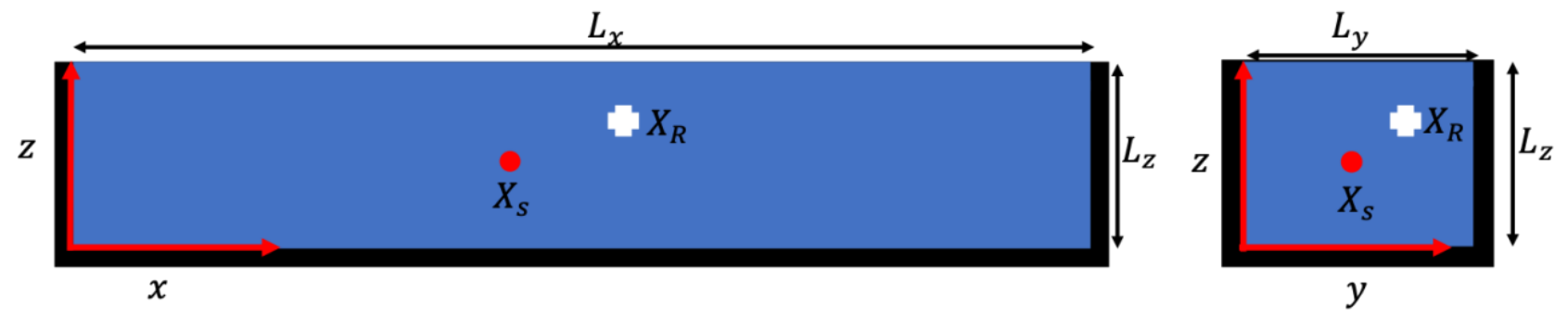

Figure 1-A Towing Tank with dimensions $L_{x}, L_{y}, L_{z}$ with source $X_{S}$ and receiver $X_{R}$

The acoustic pressure $P\left(X_{R}, \omega\right)$ at the receiver location $\boldsymbol{X}_{R}=\left(x_{r}, y_{r}, z_{r}\right)$ due to a point source at location $\boldsymbol{X}_{\boldsymbol{S}}=\left(x_{S}, y_{S}, z_{S}\right)$ is given by a summation of the pressures due to a lattice of image sources which represent reflections from the tank walls and the free surface. By simple geometry the images are placed symmetrically about each wall and free surface, forming a lattice of images out to infinity, so

$$
P\left(X_{R}, \omega\right)=\frac{j \omega \rho q}{4 \pi} \sum_{a=0}^{7} \sum_{b=0}^{\infty} A_{\underline{U}[a], \underline{L}[b]} e^{j\left[k d_{\underline{U}[i], \underline{L}[k]}\right]-\alpha(\omega) d_{\underline{U}[a], \underline{L}[b]}}
$$

where $A_{\underline{U}[a], \underline{L}[b]}$ is the pressure amplitude of the $(a, b)^{\text {th }}$ image, $\omega$ is frequency $\left(\mathrm{rad} s^{-1}\right), \rho$ is the water density, $q$ is the source volume velocity (set equal to 1 for simplicity), $k$ is wavenumber and $j=\sqrt{-1}$. Here, $\alpha(\omega)$ is the frequency-dependent volumetric absorption coefficient in nepers $/ m$, which can be calculated from an empirical formula, e.g. [5].

The location of an image source within the image lattice, is controlled through vectors $\underline{\boldsymbol{u}}$ and $\underline{\boldsymbol{l}}$, ( [4], [6], [7]), which are a given row (a,b) of matrices $\underline{\boldsymbol{U}}$ and $\underline{L}$ respectively. Figure 2 shows how $\underline{\boldsymbol{u}}$ and $\underline{\boldsymbol{l}}$ control the position of an image source. The vector $\underline{U}[a]$ in Equation 2.1 is a row of matrix $\underline{\boldsymbol{U}}$, indexed between 0 and 7 , where the entire matrix is

$$
\underline{\mathbf{U}}=\left[\begin{array}{lll}
u & v & w \\
0 & 0 & 0 \\
0 & 0 & 1 \\
0 & 1 & 0 \\
1 & 0 & 0 \\
1 & 1 & 0 \\
0 & 1 & 1 \\
1 & 0 & 1 \\
1 & 1 & 1
\end{array}\right]=\left[\begin{array}{l}
\underline{\mathbf{U}}[0] \\
\underline{\mathbf{U}}[1] \\
\underline{\mathbf{U}}[2] \\
\underline{\mathbf{U}}[3] \\
\underline{\mathbf{U}}[4] \\
\underline{\mathbf{U}}[5] \\
\underline{\mathbf{U}}[6] \\
\underline{\mathbf{U}}[7]
\end{array}\right]
$$


and $\underline{L}$ is a much larger matrix of dimensions $(\infty, 3)$, similar to [4]:

$$
\underline{L}=[l, m, n]=[\underline{\boldsymbol{L}}[b]]
$$

where $l, m, n$ each take a value between $-\infty$ and $\infty$.

In practice the summation over $b$ in Equation 2.1 is truncated according to a particular maximum image distance, and hence maximum impulse response time, chosen to achieve convergence of the tank response.

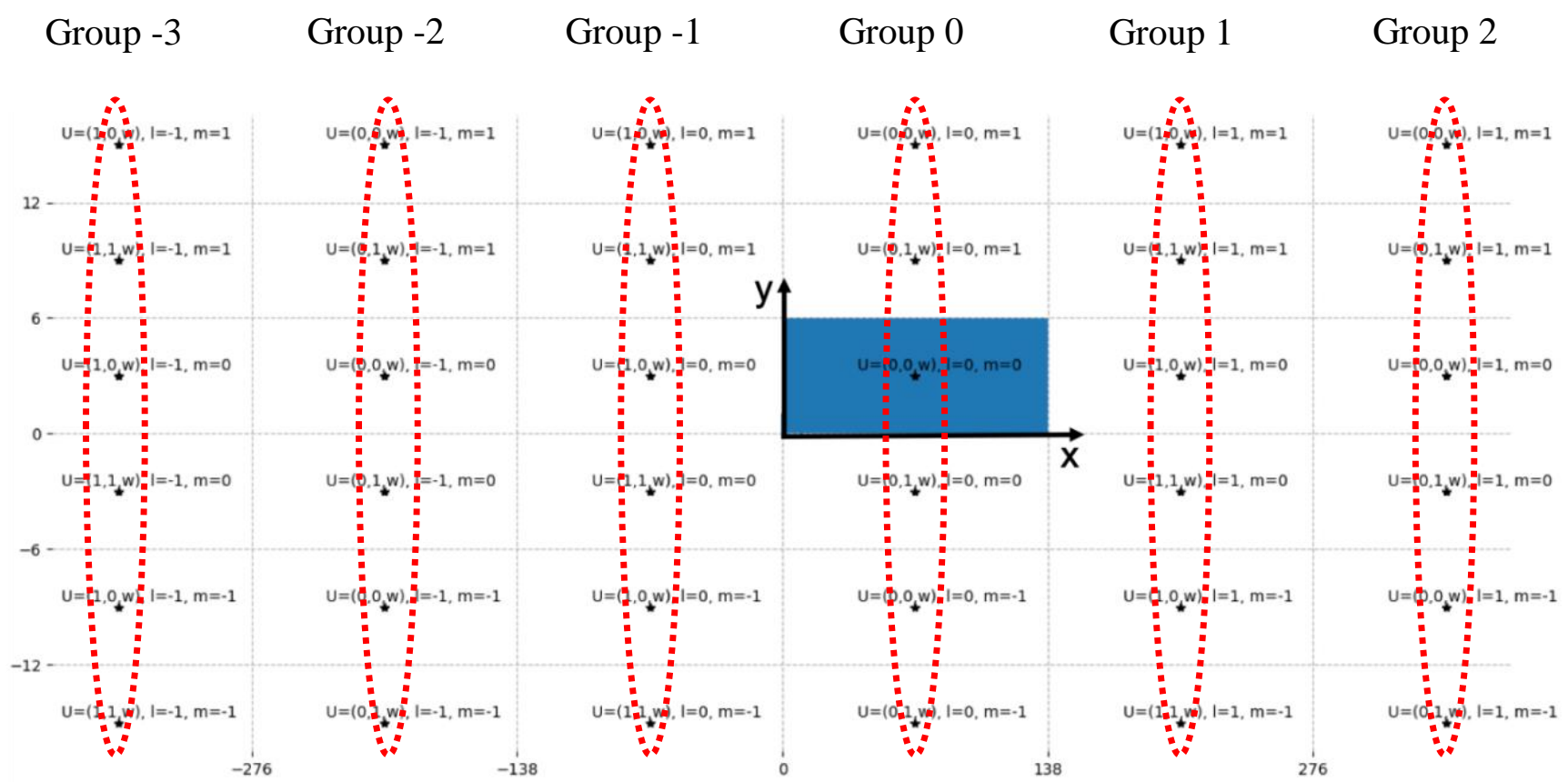

Figure 2 - Lattice of image sources in the $x$-y plane for the Boldrewood Towing Tank. Dimensions $138 x$ $6 \times 3.5 \mathrm{~m}$. Position of the image sources are controlled through matrices $\underline{\boldsymbol{U}}$ and $\underline{\boldsymbol{L}}$. Groups of image sources are highlighted in red dotted ovals. Not to scale.

The distance between a source (real or image) and the receiver is given by:

$$
d_{\underline{U}[a], \underline{L}[b]}=\left\|\left[\begin{array}{ccc}
1-2 u & 0 & 0 \\
0 & 1-2 v & 0 \\
0 & 0 & 1-2 w
\end{array}\right] \boldsymbol{X}_{s}+\left[\begin{array}{ccc}
2 l & 0 & 0 \\
0 & 2 m & 0 \\
0 & 0 & 2 n
\end{array}\right] \boldsymbol{L}_{\boldsymbol{r}}-\boldsymbol{X}_{\boldsymbol{R}}\right\|
$$

where

$$
\boldsymbol{X}_{\boldsymbol{s}}=\left[\begin{array}{c}
x_{s} \\
y_{s} \\
z_{s}
\end{array}\right], \quad \boldsymbol{X}_{\boldsymbol{r}}=\left[\begin{array}{c}
x_{r} \\
y_{r} \\
z_{r}
\end{array}\right], \quad \text { and } \mathbf{L}_{\mathbf{r}}=\left[\begin{array}{c}
\mathrm{L}_{\mathrm{x}} \\
\mathrm{L}_{\mathrm{y}} \\
\mathrm{L}_{\mathrm{z}}
\end{array}\right]
$$

The pressure amplitude $A_{\underline{U}[a], \underline{L}[b]}$ of an arbitrary image source is a function of the geometrical distance $d_{\underline{U}[i], \underline{L}[k]}$ between it and the receiver, and the number of interactions with each boundary, and is given by 


$$
A_{\underline{\underline{U}}[a], \underline{\underline{L}[b]}}=\frac{\beta_{x 1, \underline{U}[a], \underline{\underline{L}}[b]}^{|l-u|} \beta_{x 2, \underline{U}[a], \underline{\underline{L}}[b]}^{|l|} \beta_{y 1, \underline{U}[a], \underline{\underline{L}[b]}}^{|m-v|} \beta_{y 2, \underline{\underline{U}}[a], \underline{\underline{L}[b]}}^{|m|} \beta_{z 1, \underline{U}[a], \underline{\underline{L}}[b]}^{|n-w|} \beta_{z 2, \underline{U}[a], \underline{\underline{L}}[b]}^{|n|}}{d_{\underline{U}[a], \underline{L}[b]}},
$$

where, $\beta_{x 1}, \beta_{x 2}, \beta_{y 1}, \beta_{y 2}, \beta_{z 1}$ are the complex, angle-dependent plane wave reflection coefficients at the interfaces at $x=0, L_{x} y=0, L_{y}$ and $z=0$ respectively, while $\beta_{z 2}=-1$ is the reflection coefficient of the free surface.

\subsection{Plane Wave Reflection Coefficient of a Fluid-Solid Interface}

When an acoustic wave of speed $c$, is reflected at the fluid-solid interface of the tank walls, energy is lost to the solid through wave transmission into the solid, as longitudinal and shear waves, with wave speeds $c_{1}$ and $b_{1}$ respectively. The acoustic pressure reflected into the tank due to the interaction with each surface can be represented by a plane wave reflection coefficient that is a function of the incidence angle providing the images are sufficiently far from the boundary such that their wave fronts at the boundaries closely approximate to plane waves.

Assuming plane wave interaction the complex angle-dependent reflection coefficient of an arbitrary image source is given by,

$$
\beta_{\underline{U}[a], \underline{L}[b]}(\theta)=\frac{Z_{\text {in }}-Z}{Z_{\text {in }}+Z}
$$

where $Z_{\text {in }}$ is the input impedance for the solid-half space given by

$$
Z_{\text {in }}=Z_{1} \cos ^{2}\left(2 \gamma_{1}\right)+Z_{t} \sin ^{2}\left(2 \gamma_{1}\right)
$$

where the angle of incidence to the normal $\theta$, and corresponding refraction angles for compressional and shear waves, $\theta_{1}$ and $\gamma_{1}$ respectively, are related by the matching condition,

$$
k \sin (\theta)=k_{1} \sin \left(\theta_{1}\right)=k_{2} \sin \left(\gamma_{1}\right)
$$

where $k, k_{1}$ and $k_{2}$ are their respective wavenumbers,

$$
k=\omega / c, k_{1}=\omega / c_{1} \text { and } k_{2}=\omega / b_{1} .
$$

The impedance of the fluid half-space, $Z$, and the longitudinal $Z_{1}$ and shear wave impedances of the solid half-space, $Z_{1}$ and $Z_{t}$, are given by

$$
Z=\frac{\rho c}{\cos (\theta)}, \quad Z_{1}=\frac{\rho_{1} c_{1}}{\cos \left(\theta_{1}\right)} \quad \text { and } Z_{\mathrm{t}}=\frac{\rho_{1} b_{1}}{\cos \left(\gamma_{1}\right)} . \quad 2.11 a-c
$$


Note that for $\sin (\theta)>c / c_{1}$, it is necessary for $\cos \left(\theta_{1}\right)$ to be a positive imaginary quantity, making $Z_{1}$ a negative imaginary quantity [8], and hence Equation 2.7 becomes,

$$
\beta_{[a], \underline{L}[b]}(\theta)=\frac{Z_{t} \sin ^{2}\left(2 \gamma_{1}\right)-Z-j\left|Z_{1}\right| Z_{1} \cos ^{2}\left(2 \gamma_{1}\right)}{Z_{t} \sin ^{2}\left(2 \gamma_{1}\right)+Z-j\left|Z_{1}\right| Z_{1} \cos ^{2}\left(2 \gamma_{1}\right)}
$$

Similarly, for $\sin (\theta)>c / b_{1}$, Equation 2.7 becomes:

$$
\beta_{\underline{U}[a], \underline{L}[b]}(\theta)=\frac{\left|Z_{t}\right| \cos ^{2}\left(2 \gamma_{1}\right)+\left|Z_{1}\right| \sin ^{2}\left(2 \gamma_{1}\right)-j Z}{\left|Z_{t}\right| \cos ^{2}\left(2 \gamma_{1}\right)+\left|Z_{1}\right| \sin ^{2}\left(2 \gamma_{1}\right)+j Z} .
$$

The incidence angles of an image source wave approaching a given tank wall on the planes $x=0, L_{x} y=$ $0, L_{y}$ and $z=0$ are given by

$$
\theta_{x}=\operatorname{arcos}\left(\frac{\left|x_{i s}-x_{r}\right|}{d_{\underline{U}[a], \underline{L}[b]}}\right), \quad \theta_{y}=\operatorname{arcos}\left(\frac{\left|y_{i s}-y_{r}\right|}{d_{\underline{\underline{U}}[a], \underline{L}[b]}}\right) \quad \text { and } \theta_{z}=\operatorname{arcos}\left(\frac{\left|z_{i s}-z_{r}\right|}{d_{\underline{U}[a], \underline{L}[b]}}\right) . \quad 2.14 a-c
$$

where $x_{i s}, y_{i s}, z_{i s}$ is the position of the image source relative to the tank's origin given by

$$
\mathbf{X}_{\underline{\mathrm{U}}[\mathrm{i}], \underline{\mathrm{L}}[\mathrm{j}]}=\left(\left[\begin{array}{c}
\mathrm{X}_{\mathrm{i}} \\
\mathrm{y}_{\mathrm{i}} \\
\mathrm{z}_{\mathrm{i}}
\end{array}\right]\right)=\left[\begin{array}{ccc}
1-2 \mathrm{u} & 0 & 0 \\
0 & 1-2 \mathrm{v} & 0 \\
0 & 0 & 1-2 \mathrm{w}
\end{array}\right] \mathbf{X}_{\mathbf{s}}+\left[\begin{array}{ccc}
2 \mathrm{l} & 0 & 0 \\
0 & 2 \mathrm{~m} & 0 \\
0 & 0 & 2 \mathrm{n}
\end{array}\right] \mathbf{L}_{\mathbf{r}}
$$

The magnitude and phase of the reflection coefficients defined by Equation 2.8 at a fluid-solid interface for a typical concrete wall with density's and wave speeds of $\rho=1000 \mathrm{kgm}^{-3}, \rho_{1}=2400 \mathrm{kgm}^{-3}, c=$ $1482 \mathrm{~ms}^{-1}, c_{1}=3500 \mathrm{~ms}^{-1}$ and $b_{1}=1800 \mathrm{~ms}^{-1}$, can be seen in Figure 3 . 

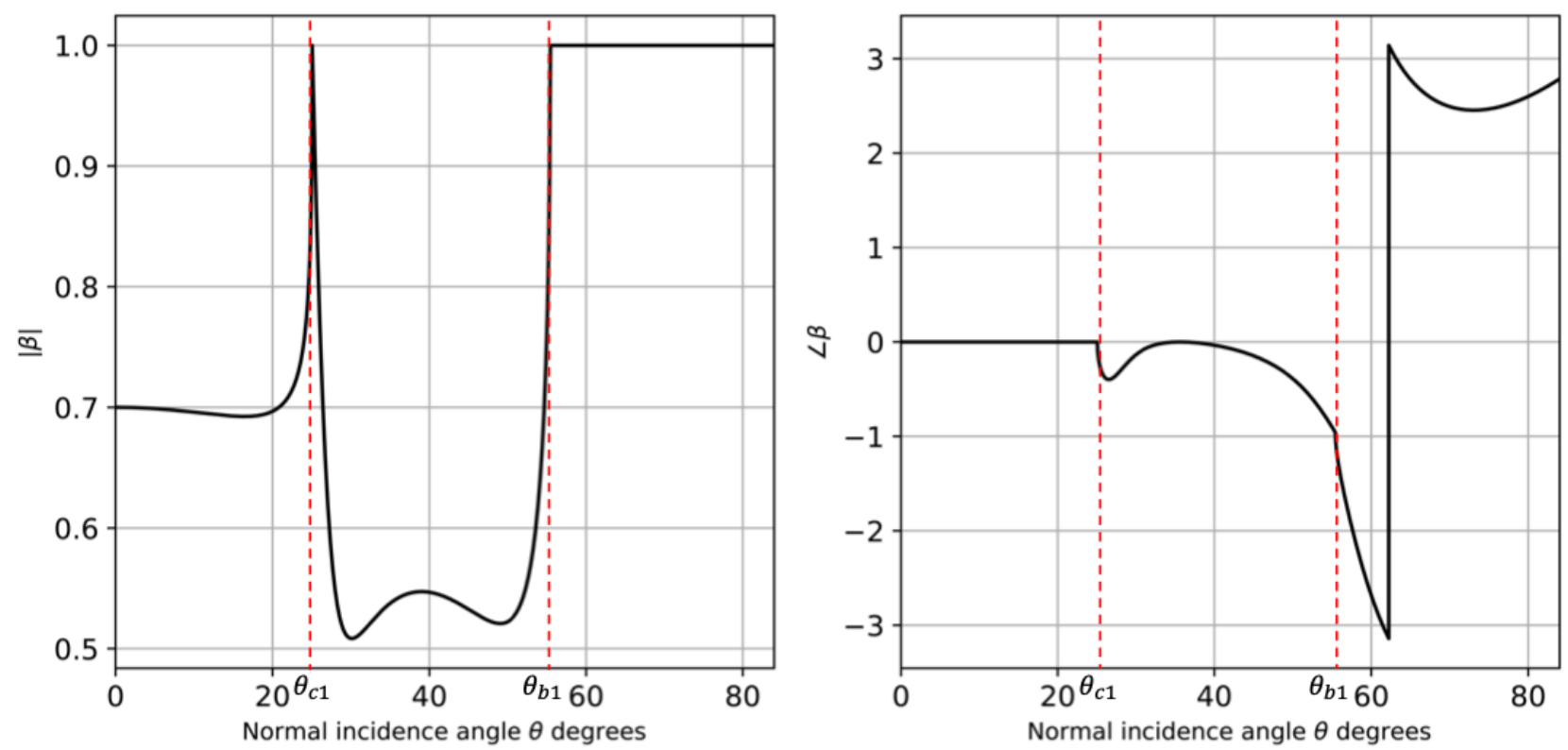

Figure 3 - Magnitude (left) and phase (right) of the reflection coefficient for waves incident on a waterconcrete interface. $\rho=1000 \mathrm{kgm}^{-3} \rho_{1}=2400 \mathrm{kgm}^{-3}, c=1482 \mathrm{~ms}^{-1}, c_{1}=3500 \mathrm{~ms}^{-1}$ and $b_{1}=1800 \mathrm{~ms}^{-1}$. $\theta_{c 1} \approx 25^{\circ}, \theta_{b 1} \approx 55^{\circ}$.

The reflection coefficient shown in Figure 3 is characterised by two critical angles $\sin \left(\theta_{c 1}\right)=c / c_{1}$ and $\sin \left(\theta_{b 1}\right)=c / b_{1}$ associated with the compressional and shear wave speeds in the solid respectively. Total internal reflection is predicted at $\theta=\theta_{c 1}$ and for $\theta>\theta_{b 1}$ at which sound is reflected back into the tank with unit reflection coefficient.

At the first critical angle, $\theta=\theta_{c 1}$, transmitted longitudinal waves propagate along the solid - fluid interface (lateral waves). Above the first critical angle, the transmitted longitudinal wave is evanescent. Once the second (shear) critical angle has been reached, $\theta=\theta_{b 1}$, total internal reflection occurs for a range of incidence angles. Although the incidence angle for reflections between two parallel walls remains constant, the angle of incidence from subsequent interactions with other perpendicular walls are likely to be less than this critical angle, leading to energy loss from the water.

One of the potential limitations of the Image Source Model presented above is the use of plane wave reflection coefficients to predict the contribution of the various image sources. Clearly this approximation is accurate for the image sources many wavelengths from the receiver whose wave fronts at the tank boundaries closely approximate plane waves. However, this approximation may lead to significant errors for those images that are closest to the receiver which require allowance for the interaction of the curved wave fronts with the plane boundaries. Furthermore, the inclusion of spherical wave fonts also allows the effect of head waves to be predicted, which propagate along the fluid - solid interface and re-radiate into the tank at the critical angle. The next section is concerned with the exact computation of the acoustic field due to the first order image sources, taking into account the interaction of the spherical wave fronts with the tank boundaries. By comparing these predictions with those obtained using the plane wave reflection the effects of wave front curvature may be assessed. 


\subsection{Reflection of a Spherical Wave at a Fluid-Solid Interface}

An incident spherical wave of the form $\exp (j k R) / R$ can be represented by the integration over a continuum of plane wave components in the wavenumber domain, where the reflection of each plane wave can be calculated from the plane wave reflection at their respective interaction angles with the plane boundary, as can be seen in Figure 4. Here, we present only the final expression for the reflected acoustic pressure $P$ at, due to a spherical wave reflecting from a plane boundary which has an angle dependent plane wave reflection coefficient $\beta(\theta)$. The geometry for the derivation of this equation is seen in Figure 5.

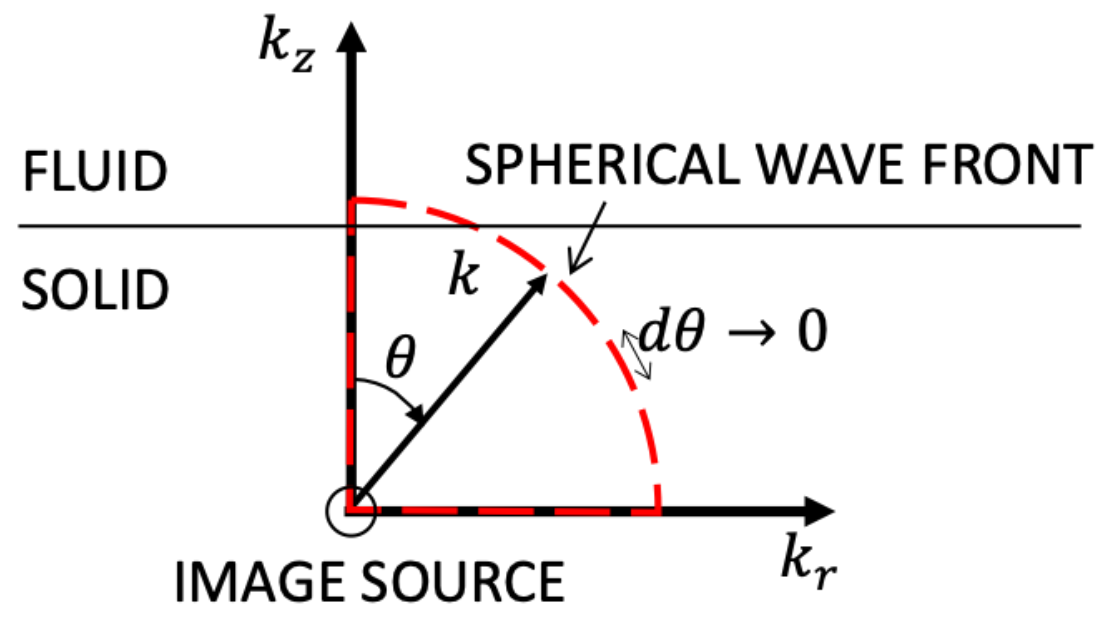

Figure 4 - A spherical wave front of an image source, represented as an infinite number of plane waves in the wavenumber domain.

The reflected acoustic pressure $P$ at an observer location $z_{r}$ of unit amplitude at height $z_{s}$ above the boundary and horizontal distance $r$ from the observer above a plane boundary with plane wave reflection coefficient $\beta(\theta)$ is ([8], [9])

$$
P\left(k r, k z_{r}\right)=j k \oint_{0}^{\frac{\pi}{2}-j \infty} J_{0}(k r \sin (\theta)) e^{j k\left(z_{S}+z_{r}\right) \cos (\theta)} \beta(\theta) \sin (\theta) d \theta
$$

where $J_{0}$ is the Bessel function of zero order, $k$ is the wave number and $r=\sqrt{x^{2}+y^{2}}$. Equation 2.16 makes explicit that the reflection pressure is only a function of non-dimensional distances $k r$ and $k z_{r}$ (i.e., distance compared to the acoustic wavelength). Equation 2.16 specifies a path of integration shown in Figure 6 , where the range of $\theta$ between 0 and $\pi / 2$ correspond to the continuum of propagating wave components while imaginary contribution between $\pi / 2$ and $\pi / 2$-j $\infty$ represent inhomogeneous decaying components of the acoustic field, which constitute the source near field. 
Although the contour of integration in Equation 2.16 on the complex $\theta$ plane is to infinity, convergence to 0 is achieved at an upper limit of $a$ given by,

$$
a_{\text {lim }}=\operatorname{arcsinh}\left(\frac{200}{k z_{t}}\right)
$$

as the exponential term in Equation 2.16 decays to 0 at $a_{\text {lim }}$.

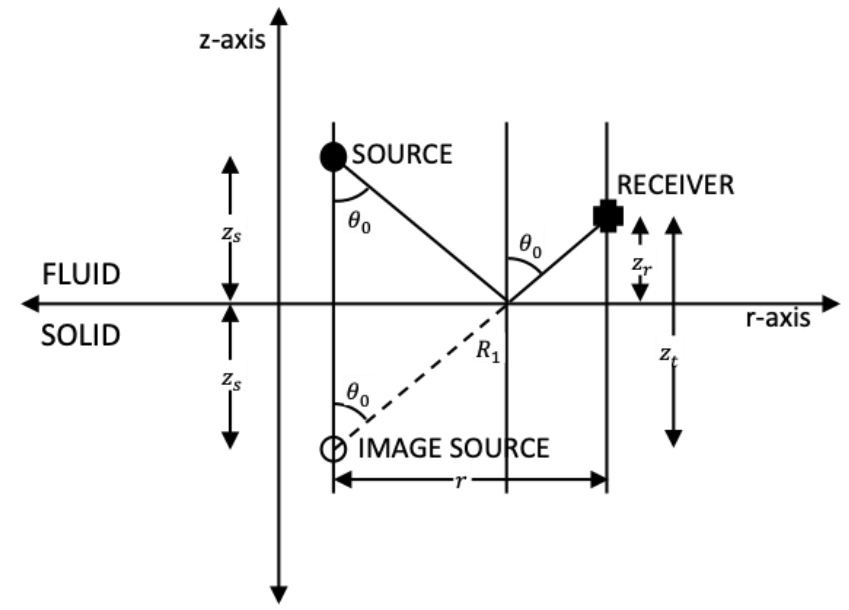

Figure 5 - Geometry for the plane wave integration in the $z$-r plane, where, $d z=z_{r}+$ $z_{s}=z_{t}$.

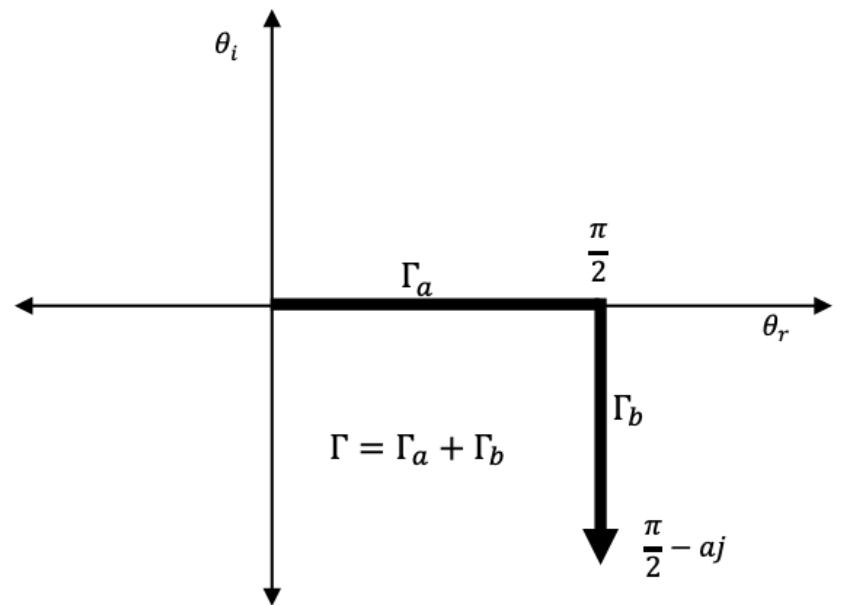

Figure 6-Contour of integration in the complex $\theta$-plane for the reflected field.

Using Cauchy's integral theorem, the contour of integration $\Gamma$ can be split into the two paths $\Gamma_{A}$ and $\Gamma_{B}$ where for the integration over $\Gamma_{A}, \theta$ is purely real up to $\frac{\pi}{2}$ and over $\Gamma_{B}, \frac{\pi}{2}+0 j \leq \theta \leq \frac{\pi}{2}-a j$. Equation 2.16 can now be rewritten as

$$
P\left(k r, k z_{t}\right)=k\left[j \int_{0}^{\frac{\pi}{2}} A(\theta) d \theta+\int_{0}^{a_{l i m}} B(a) d a\right]
$$

where

$$
A(\theta)=J_{0}(k r \sin (\theta)) e^{j k z_{t} \cos (\theta)} \sin (\theta) \beta(\theta)
$$

and

$$
B(a)=J_{0}(k r \cosh (a)) e^{-k z_{t} \sinh (\mathrm{a})} \beta\left(\frac{\pi}{2}-j a\right) \cosh (a),
$$


which is now in a form more suitable for numerical integration. Note that the integrands are highly oscillatory functions and therefore adaptive routines, such as Scipy's quad function [10], must be used to obtain accurate solutions.

At a given complex normal incidence angle along integration path $\Gamma_{B}$, the reflection coefficient of Equation 2.7, can become infinite as the denominator becomes 0 . This is known as the Stonely pole [11], and causes a singularity in the integral of Equation 2.17. To push the Stonely pole away from the integration path, a small negative imaginary part can be added to the shear wave speed $b_{1}$.

\subsection{Effective spherical wave reflection coefficient}

The image source formulation of Equation 2.1 for the acoustic field can be used directly to include the effects of spherical wave interaction with the boundaries for first order images, by the use of an effective spherical wave reflection coefficient $\beta_{S}\left(k r, k z_{t}\right)$ defined by

$$
P\left(k r, k z_{t}\right)=\beta_{S}\left(\theta_{0}, k z_{t}\right) \frac{e^{-j k R_{1}}}{R_{1}}
$$

where $P\left(k r, k z_{t}\right)$ is computed from Equation 2.18, and $R_{1}=\sqrt{r^{2}+z_{t}^{2}}$. The spherical wave reflection coefficient is now a function of normal incidence angle $\theta_{0}=\arctan r / z_{t}$ and frequency. First order images are those which reflect once off a single wall, defined through $\underline{U}[i], \underline{L}[k]$ by Table 1 . The geometry of Figure 5, is simply re-orientated appropriately for the $\mathrm{x}$ and y plane walls. The inclusion of spherical wave reflection coefficients for first order images is now a first order correction to the Image Source Model. The first order image above the free surface remains as a plane wave image, as the pressure release boundary condition is independent of angle.

Table 1- First order images of each tank wall defined by vectors $(l, m, n)$ and $(u, v, w)$

\begin{tabular}{c|ccc|ccc} 
Wall & $\boldsymbol{l}$ & $\boldsymbol{m}$ & $\boldsymbol{n}$ & $\boldsymbol{u}$ & $\boldsymbol{v}$ & $\boldsymbol{w}$ \\
\hline$x 1$ & 0 & 0 & 0 & 1 & 0 & 0 \\
\hline$x 2$ & 1 & 0 & 0 & 1 & 0 & 0 \\
\hline$y 1$ & 0 & 0 & 0 & 0 & 1 & 0 \\
\hline$y 2$ & 0 & 1 & 0 & 0 & 1 & 0 \\
\hline$z 1$ & 0 & 0 & 0 & 0 & 0 & 1 \\
\hline$z 2$ (free surface) & 0 & 0 & 1 & 0 & 0 & 1
\end{tabular}

By way of example, $\beta_{S, z}\left(\theta_{0}, k z_{t}\right)$ is calculated for four different values of $k z_{t}$ (where $z_{t}=z_{s}+z_{r}$ ) for the bottom parameters used to compute Figure 3. The magnitude and phase of $\beta_{S}$ are plotted in Figure 7 versus incidence angle and compared against the plane wave reflection coefficient, plotted as a solid line. Naturally, spherical wave effects deviate most from plane wave behaviour for small values of $k z$. Oscillations in the spherical wave reflection coefficients can be observed that are not apparent in the plane wave reflection coefficient and are caused by interference between the totally reflected field and the two lateral (head) wave fields [9] which travel along the fluid-solid interface and then re-radiate back to the fluid. This is visually shown in Figure 8, as the grey zone where the re-radiated lateral wave field 
is shown propagating away from the interface at the critical angle $\left(\theta_{c r, c 1}\right.$ or $\left.\theta_{c r, b 1}\right)$. Note that for high grazing angles, when the receiver is not located inside the lateral wave field zone (receiver 1), only a partially reflected wave exists. At receiver position 2, however there are interference contributions to the acoustic field by both total internally reflected waves and lateral waves.
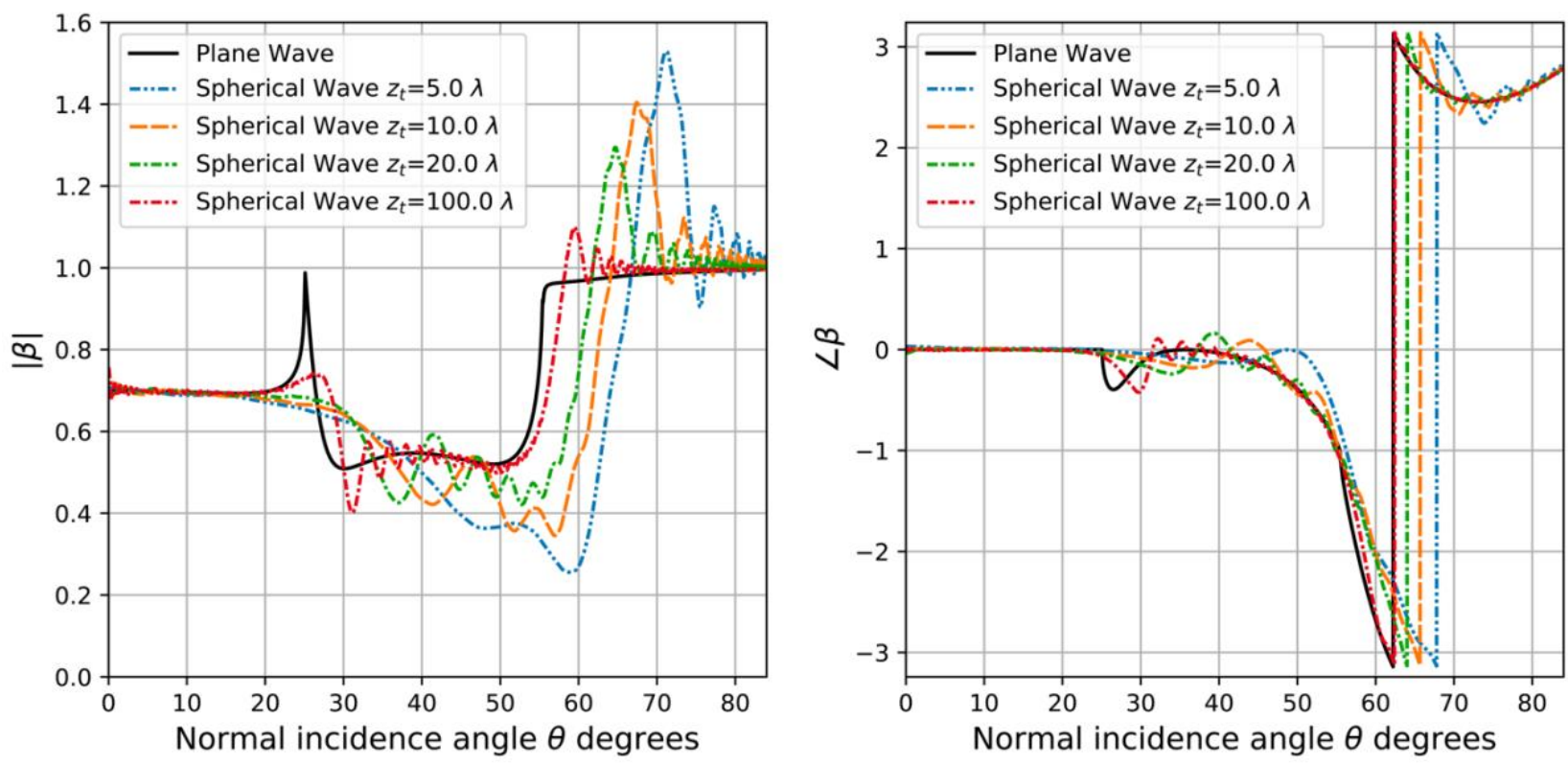

Figure 7 Magnitude (left) and phase (right) of the reflection coefficient for plane and spherical waves incident on a plane interface. The interface has properties $\rho=1000 \mathrm{kgm}^{-3} \rho_{l}=2400 \mathrm{kgm}^{-3}, c=1482 \mathrm{~ms}^{-}$ ${ }^{1} c_{1}=3500 \mathrm{~ms}^{-1}$, and $b_{1}=1800-1 \mathrm{jss}^{-1}$ (imaginary part to shear wave added to remove the Stonely pole from contour of integration). $\lambda$ is the wavelength emitted by the source in $m, z_{t}$ is the vertical distance between image source and receiver as defined in Figure 5. 


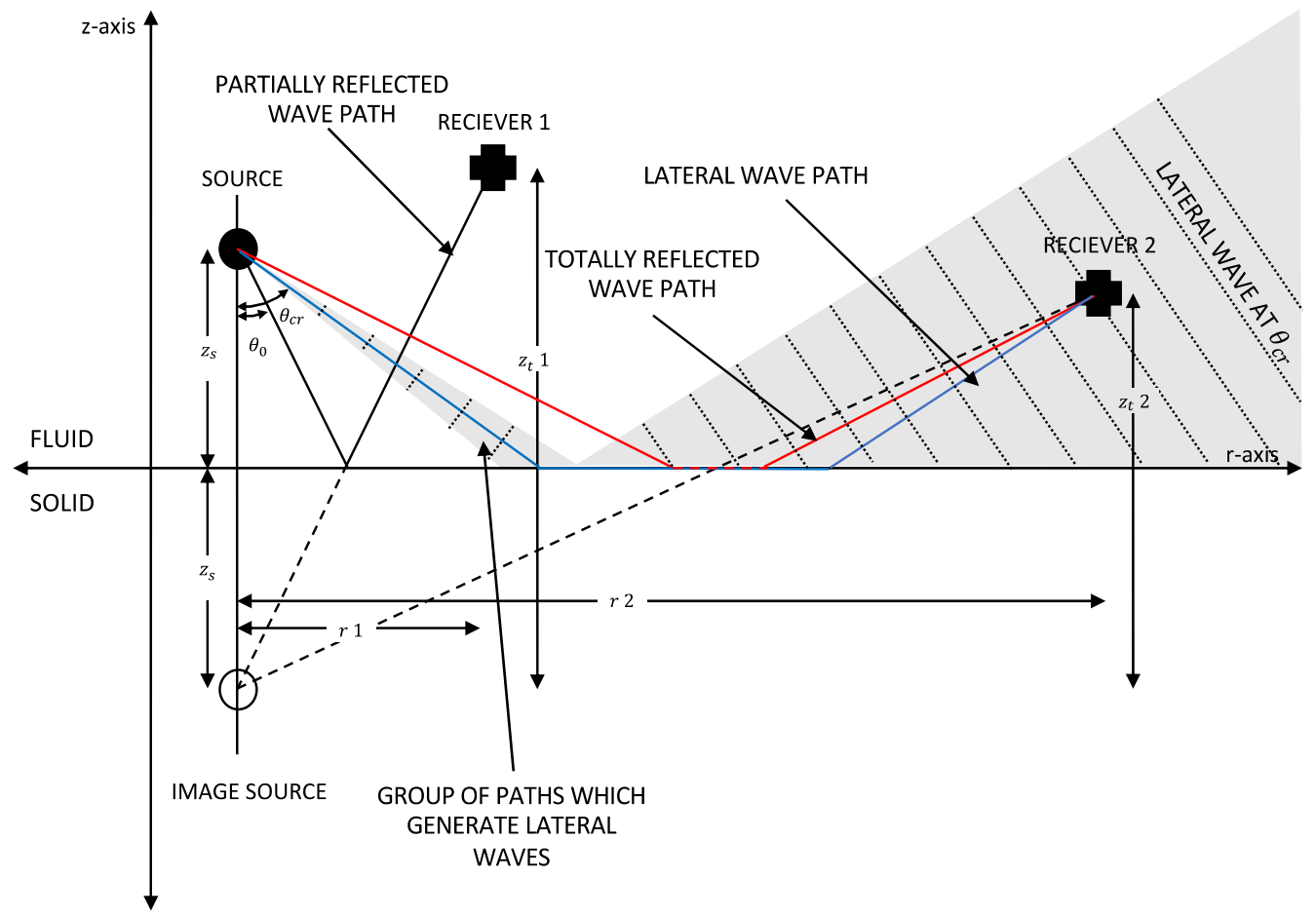

Figure 8 - Reflected field from a fluid-solid interface, indicating the existence of totally internally reflected waves and head waves. Adapted from [9].

The difference between the Sound Pressure Level, due to a reflected spherical wave $\left(S P L_{S}\right)$ and reflected plane wave $\left(S P L_{P}\right)$ at a receiver, is given by the ratio between the spherical $\left(\beta_{S}\right)$ and plane wave $\left(\beta_{S}\right)$ reflection coefficients:

$$
S P L_{S}-S P L_{P}=20 \log \frac{\beta_{S}\left(\theta_{0}, k z_{t}\right)}{\beta\left(\theta_{0}\right)} \mathrm{dB} .
$$

The ratio of the spherical to plane wave reflection coefficients (Equation 2.22) for increasing $k z_{t}$ and $\theta_{0}$, for the wall parameters used in Figure 7, can be seen in Figure 9. For small $\theta_{0}$ (low $r$ ), the spherical wave reflection coefficient converges to the plane wave result with increasing $k z_{t}$, as at this $r$ there exists only a partially reflected wave, with the effects of wave curvature decreasing with large $k z_{t}$. Convergence is also seen at high incidence angles, beyond the shear wave critical angle, for high $k z_{t}$, as the surface wave propagating along the interface is strongly attenuated, relative to the total internally reflected waves. Convergence, however is not achieved for ranges $r$, at which $\theta_{0}$ is close to the critical angles $\theta_{c r, c 1}$ and $\theta_{c r, b 1}$, due to the development of a caustic, where total internally reflected and lateral waves coalesce into one [9].

It is therefore recommended that the first order images in the Image Source Model use the spherical wave reflection coefficient for frequencies where $z_{t}<240 \lambda\left(k z_{t}<1500\right)$, and additionally when the receiver is located at ranges close to the lateral wave field $\left(r=z_{t} \tan \theta_{c 1, b 1}\right)$. 


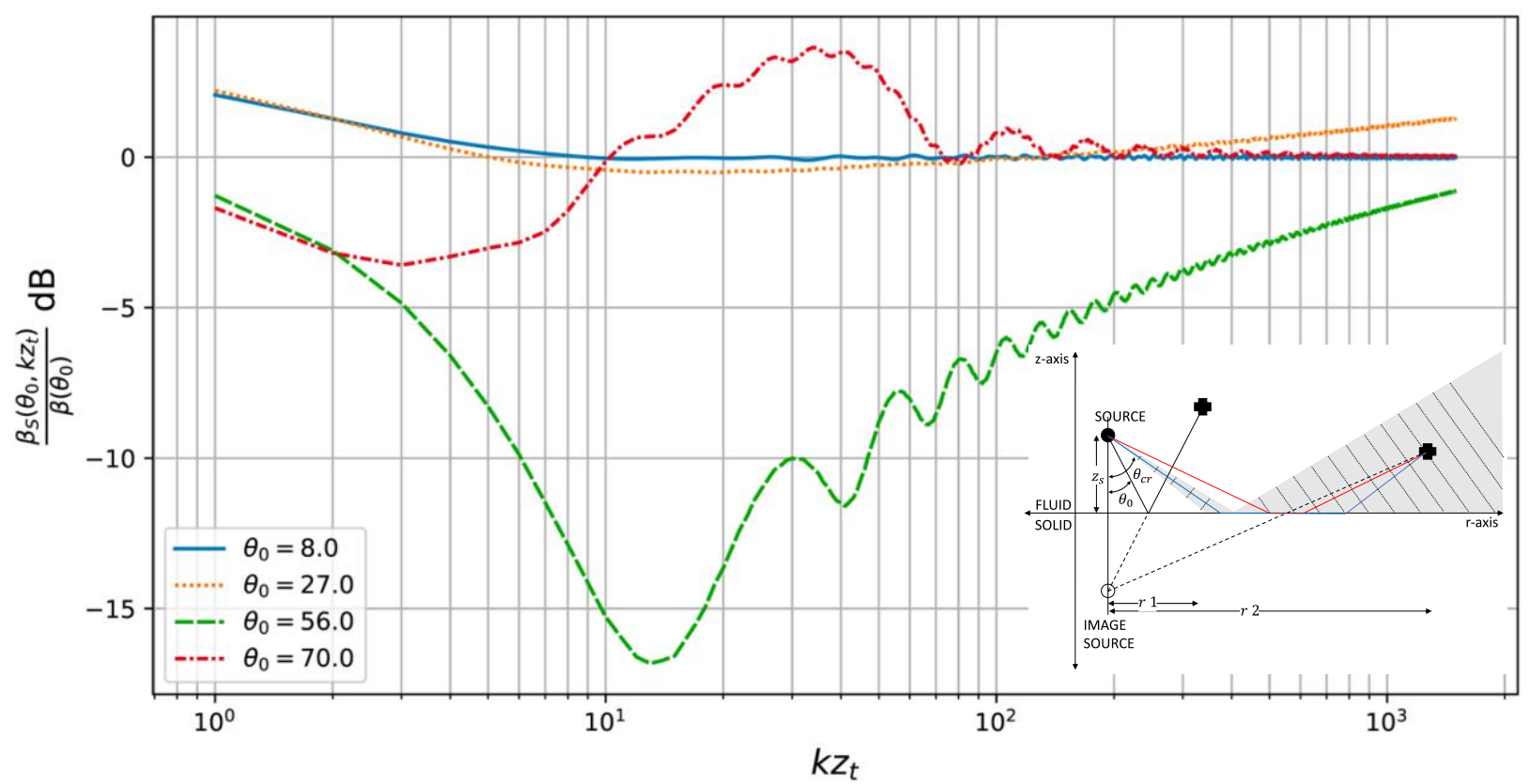

Figure 9 Ratio of spherical to plane wave reflection coefficients with increasing $k z_{t}$ for various normal incidence angles $\theta_{0}$. Note that an increase in $\theta_{0}$ is an increase in horizontal range $r$, as defined in Figure 5. $\theta_{c 1} \approx 25^{\circ}, \theta_{b 1} \approx 55^{\circ}$.

\subsection{Spherical wave effects on the tank acoustic field}

To illustrate clearly the spherical wave effects on the acoustic response of a towing tank, the acoustic field was computed using the image model formulation of Equation 2.1, for the $1^{\text {st }}$ order images described in Table 1, using the plane wave approximation and with the spherical wave correction. The correction was applied for each of the first order images at each interface for frequencies where $k z_{t}<1500$, where $z_{t}$ is the perpendicular distance, relative to the interface orientation, between image source and receiver.

The tank, assumed at be similar to that of the tank at Boldrewood at the University of Southampton, has dimensions of $L_{x}, L_{y}$ and $L_{z}=138 \mathrm{~m}, 6 \mathrm{~m}$ and $3.5 \mathrm{~m}$ and concrete walls with $\rho=3500 \mathrm{~kg} / \mathrm{m}-, c_{1}=3500 \mathrm{~m} / \mathrm{s}$ and $b_{1}=1800 \mathrm{~m} / \mathrm{s}$. The source was located at $\mathbf{X}_{\mathbf{s}}=(69,2.76,3.14)$ with receiver at $\mathbf{X}_{\mathbf{r}}=(69+r$, $2.76,3.14)$.

The difference in predicted Sound Pressure Level between the spherical wave correction $\left(S P L_{S}\right)$ and the plane wave approximation $\left(S P L_{P}\right)$ is shown in Figure 10, for ranges $r=0.5,2.0,4.0$ and $6.0 \mathrm{~m}$. Large differences $(> \pm 5 \mathrm{~dB})$ at modal and antimodel frequencies are observed at high frequencies, whereas at low frequencies, there is a large frequency range with a positive $S P L$ difference greater than $5 d B$. The differences vary significantly with increasing range as the receiver transverses into the lateral wave field for the $y$ and $z$ plane walls. For the $x$ plane walls, there are a relatively low number of frequencies which need the spherical wave correction as " $k z_{t}$ " ( $k x_{t}$ relative to tank origin) is large and $r$ is small, which means the wave front has very little curvature and is well approximated by a plane wave. 

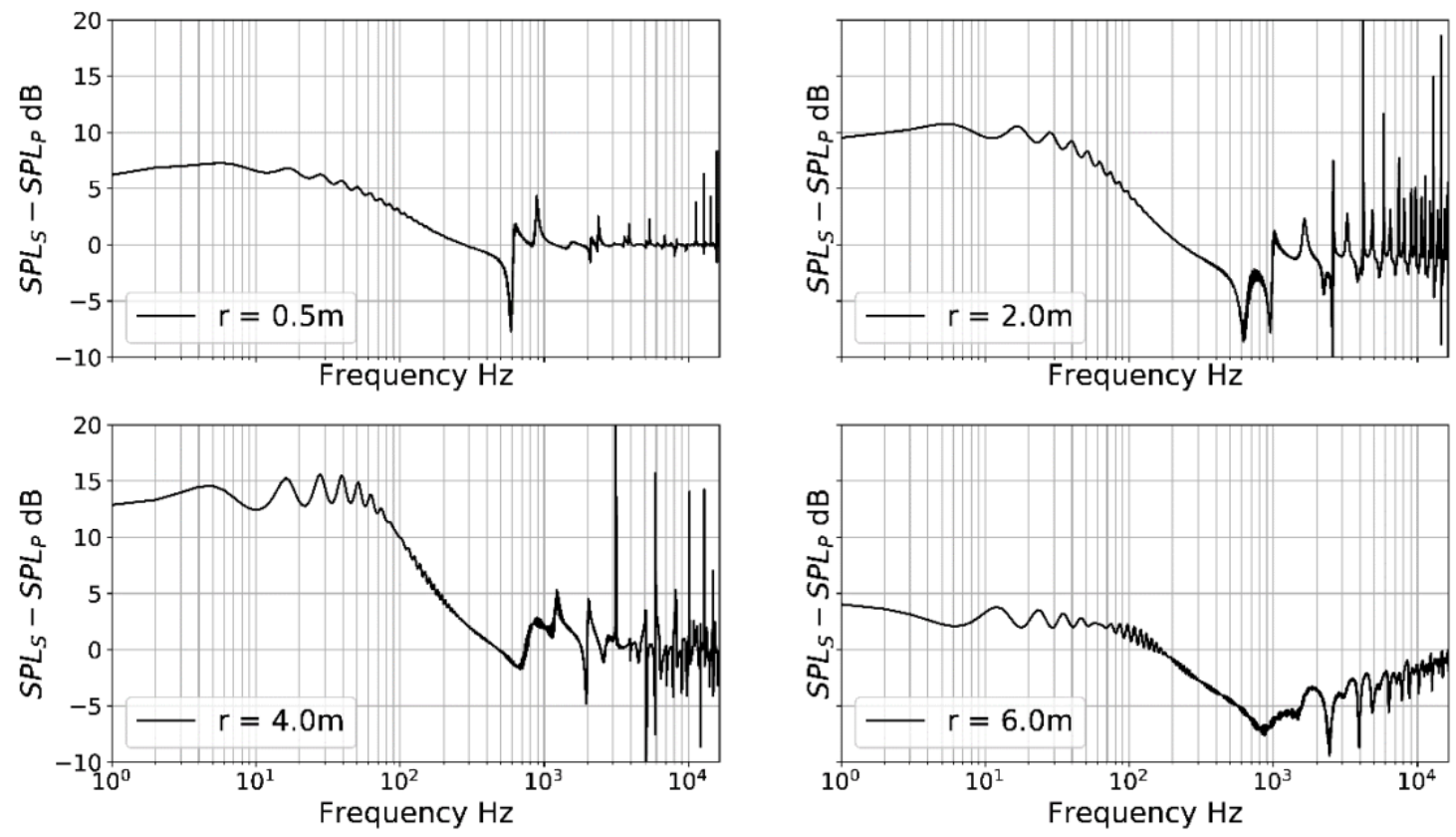

Figure 10 Difference between total sound pressure levels $S P L_{S}$ and $S P L_{P}$ in the Boldrewood towing tank, due to the $1^{\text {st }}$ order images, predicted with spherical and plane wave reflection coefficients.

\section{Comparison with experiment}

Here we validate predictions obtained from the adapted Image Source Model by comparison with published measurements of the transfer function between acoustic pressure measurements at two positions in the Depressurized Wave Basin [2] at the Maritime Research Institute Netherlands (MARIN). The results are also compared to the analytical solutions in free field and beneath a free surface to establish the relative importance of the free field radiation and the Lloyd's mirror effect. The simulation was undertaken with a frequency interval $\Delta f=1 \mathrm{~Hz}$ up to $65 \mathrm{kHz}$. The measurements from [2] were undertaken with $f_{s}=130 \mathrm{kHz}$, using a repeating log-chirp excitation signal between $710 \mathrm{~Hz}$ to $22.72 \mathrm{kHz}$.

The tank is approximated to have perfectly rectangular geometry with concrete walls. However, the Depressurized Wave Basin has chamfered corners between the bottom and side walls, as well as 200 aluminium wave paddles, running along $120 \mathrm{~m}$ of a side wall.

The simulated transfer function, between the pressure at a reference $\left(P_{\text {ref }}(\omega)\right)$ and receiver $\left(P_{\text {rec }}(\omega)\right)$ hydrophone, was calculated from Equation 3.1, using the Image Source model describe in section 2, with $1^{\text {st }}$ order spherical wave corrections. The measured transfer function was calculated using the auto correlation $S_{P_{\text {rec }} P_{r e c}}(\omega)$ and cross correlation $S_{P_{\text {rec }} P_{r e f}}(\omega)$ of the measured time domain signal. $S_{P_{\text {rec }} P_{\text {rec }}}(\omega)$ and $S_{P_{\text {rec }} P_{\text {ref }}}(\omega)$ are calculated using the Welch's method [12] with a window length equal to the duration of $1 \log$ chirp. See configuration $D$ of [2] for the details of the experimental setup. A summary of the setup can be seen in Table 2 . 
Table 2 Locations of Source, reference and receiver hydrophones for measurements in the Depressurized Wave Basin from [2]

\begin{tabular}{|c|c|c|c|c|c|c|c|c|}
\hline \multicolumn{3}{|c|}{ Source Location } & \multicolumn{3}{c|}{ Reference Hydrophone } & \multicolumn{3}{c|}{ Receiver Hydrophone } \\
\hline $\boldsymbol{x}_{\boldsymbol{s}}$ & $\boldsymbol{y}_{\boldsymbol{s}} \mathbf{m}$ & $\begin{array}{c}\boldsymbol{L}_{\boldsymbol{z}}-\boldsymbol{z}_{\boldsymbol{s}} \\
\mathbf{m}\end{array}$ & $\boldsymbol{x}_{\boldsymbol{r}} \mathbf{m}$ & $\boldsymbol{y}_{\boldsymbol{r}} \mathbf{m}$ & $\begin{array}{c}\boldsymbol{L}_{\boldsymbol{z}}-\mathbf{z}_{\boldsymbol{r}} \\
\mathbf{m}\end{array}$ & $\boldsymbol{x}_{\boldsymbol{r}} \mathbf{m}$ & $\boldsymbol{y}_{\boldsymbol{r}} \mathbf{m}$ & $\begin{array}{c}\boldsymbol{L}_{\boldsymbol{z}}-\mathbf{z}_{\boldsymbol{r}} \\
\mathbf{m}\end{array}$ \\
\hline 120 & 6.512 & 0.36 & 118.8 & 6.512 & 0.36 & 118 & 6.512 & 1.5 \\
\hline
\end{tabular}

Further validation was undertaken in the time domain through calculating the Impulse Response (Equation 3.2) from the Transfer Function:

$$
\begin{gathered}
H(\omega)=\frac{P_{r e c}(\omega)}{P_{r e f}(\omega)}=\frac{S_{P_{\text {rec }} P_{r e c}}(\omega)}{S_{P_{r e c} P_{r e f}}(\omega)} \\
\text { and } \mathrm{h}(\mathrm{t})=\operatorname{ifft}[\mathrm{H}(\omega)] .
\end{gathered}
$$

\subsection{Transfer Function and Impulse Response}

It is well known that the dominant response to a shallow-water acoustic source is governed by the Lloyd's Mirror effect, which is clearly observed in Figure 11 for both simulated (bottom) and measured (top) transfer functions. However, these high frequency-resolution transfer functions reveal high levels of fluctuation arising from interference due to axial resonances. The frequency variability in Figure $11 \mathrm{can}$ be seen to fluctuate on three different scales; large scale due to Lloyd's mirror interference $\left(\Delta f_{1}\right)$, medium scale $\left(\Delta f_{2}\right)$ due to transverse ( $y, z$-directions) modes and small scale $\Delta f_{3}$ due to longitudinal ( $x$-direction) modes. These three scales have been labelled 1,2 and 3 consecutively on Figure 11, and can be approximated by:

$$
\Delta f_{1} \sim \frac{c}{r_{2}-r_{1}}
$$

where $r_{2}$ and $r_{1}$ are the distances between the first order image above the free surface to receiver and direct field distance, respectively. The medium scale interval is given by

$$
\Delta f_{2} \sim \frac{c}{L_{y}} \sim \frac{c}{2 L_{z}}
$$

where $L_{y}$ and $L_{z}$ are the width and depth of the tank respectively. And the fine scale by

$$
\Delta f_{3} \sim \frac{c}{L_{x}},
$$

where $L_{x}$ is the length of the tank and $c$ is the speed of sound. 
Additionally, from Figure 11, it can be seen that the measured transfer function is not perfectly aligned with the dominating Lloyd's Mirror transfer function. This is possibly due to uncertainty in the measurement of the hydrophone and source locations in the tank from [2]. This is further evident in the Impulse Response shown Figure 12, where a consistent time-shift between measured and simulated arrival times is observed.
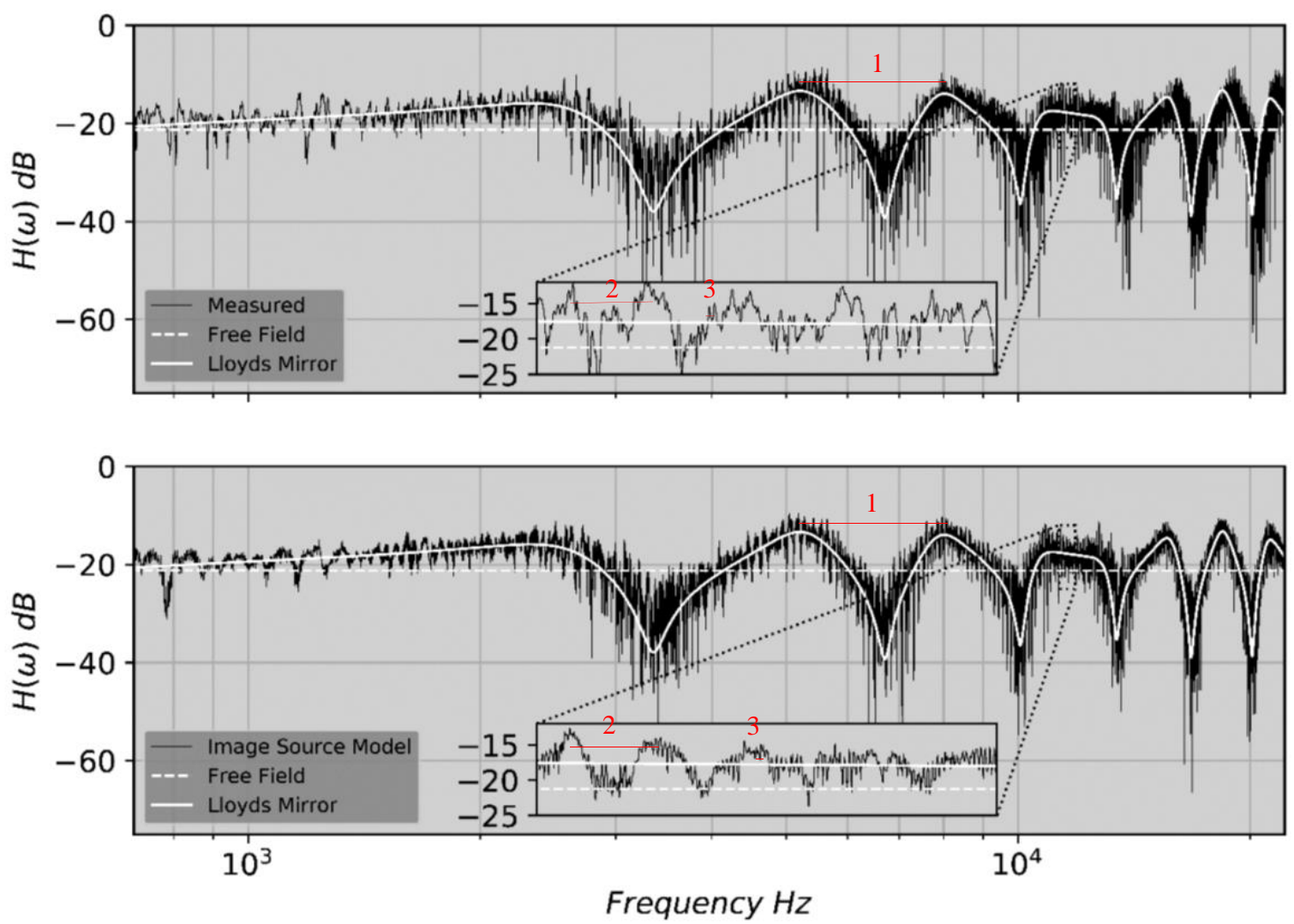

Figure 11 A comparison between measured (top) and simulated (bottom) transfer functions between reference and receiver hydrophones. The locations are given in Table 2. Overlaid are the analytical free field and Lloyd's mirror transfer functions in white. 


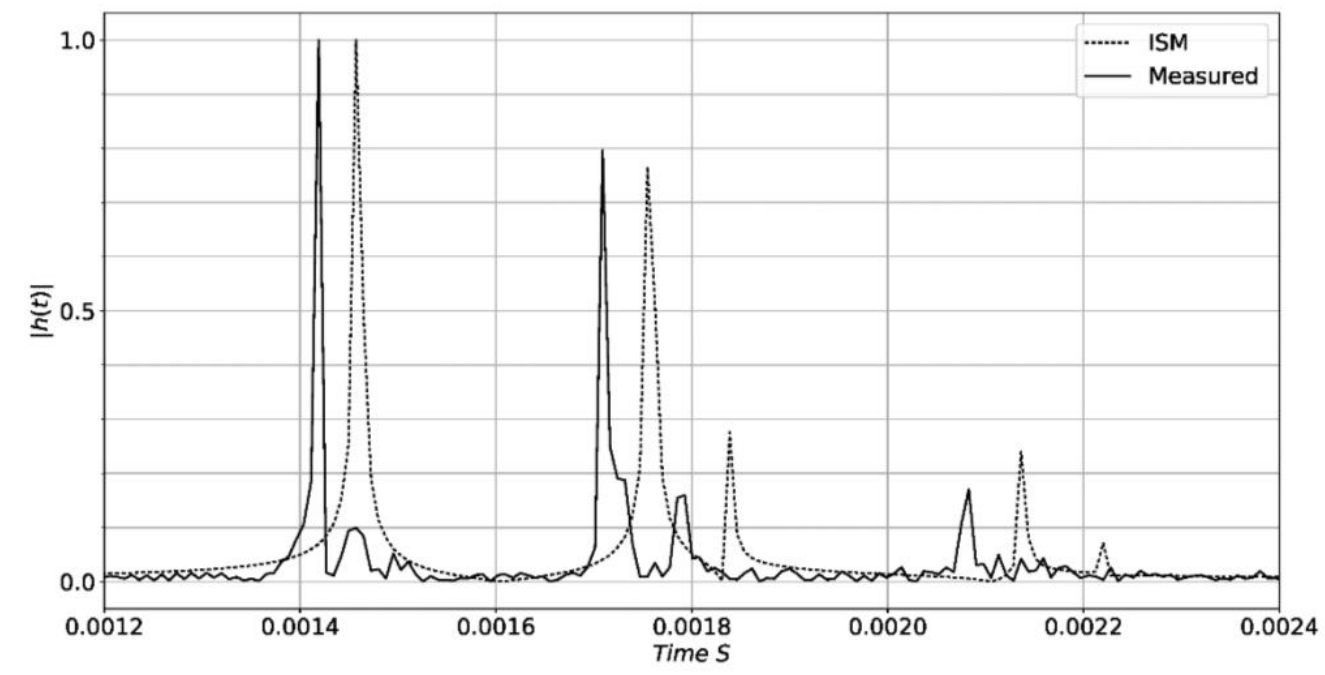

Figure 12 Normalised impulse response calculated from the ifft of the transfer functions in Figure 11. Solid line is from measurements and dotted line from Simulated Image Source Model (ISM).

\section{Comparison of Towing Tanks}

Now that the Image Source Model has been validated, it is used to calculate the reverberation time and reverberation distance of three different sized tanks. The dimensions of the three towing tanks are shown in Table 3, together with the three scales of expected frequency periodicity predicted by Equations 3.3 to 3.5 .

To isolate the effect of tank size, the positions of the source, reference and receiver hydrophones were selected for each tank, to be a scaled position relative to the measurements in the Depressurized Wave Basin, however the depth under the free surface was kept at $0.36 \mathrm{~m}$.

Table 3 Tank Dimensions and fundamental mode frequencies assuming rigid walls

\begin{tabular}{|l|c|c|c|c|}
\hline \multicolumn{1}{|c|}{ Tank Name } & $\begin{array}{c}\text { Dimensions } \\
\boldsymbol{L}_{\boldsymbol{x}}, \boldsymbol{L}_{\boldsymbol{y},} \boldsymbol{L}_{\boldsymbol{z}} \\
{[\mathbf{m}]}\end{array}$ & $\begin{array}{c}\text { Small Scale } \\
\Delta f_{3} \\
{[\mathbf{H z}]}\end{array}$ & $\begin{array}{c}\text { Medium Scale } \\
\Delta f_{2} \\
{[\mathbf{H z}] .}\end{array}$ & $\begin{array}{c}\text { Large Scale } \\
{\left[f_{1}\right.} \\
{[\mathbf{H z}]}\end{array}$ \\
\hline $\begin{array}{l}\text { Depressurized } \\
\text { Wave Basin }\end{array}$ & $240,15.7,7.85$ & 3.0 & $45.6,45.8$ & 3350 \\
\hline $\begin{array}{l}\text { Boldrewood } \\
\text { Towing Tank }\end{array}$ & $138,6.0,3.5$ & 5.2 & 120,103 & 2019 \\
\hline $\begin{array}{l}\text { Solent Towing } \\
\text { Tank }\end{array}$ & $60,3.7,1.8$ & 12 & 195,200 & 3690 \\
\hline
\end{tabular}

Table 4 Source, Reference and Receiver Hydrophone locations

\begin{tabular}{|c|c|c|c|c|c|c|c|c|c|}
\hline Tank Name & Sourc & cation & $\mathrm{y}, \mathrm{z}[\mathrm{m}]$ & $\begin{array}{l}\text { Refere } \\
{[\mathrm{m}]}\end{array}$ & Loc: & $\mathbf{x}, \mathbf{y}, \mathbf{z}$ & $\begin{array}{l}\text { Recei } \\
{[\mathrm{m}]}\end{array}$ & Loca & $\mathbf{x}, \mathbf{y}, \mathbf{z}$ \\
\hline $\begin{array}{l}\text { Depressurized } \\
\text { Wave Basin }\end{array}$ & 120 & 7.21 & 7.49 & 119.8 & 7.21 & 7.49 & 118 & 7.21 & 6.35 \\
\hline
\end{tabular}




\begin{tabular}{|l|c|c|c|c|c|c|c|c|c|}
\hline $\begin{array}{l}\text { Boldrewood } \\
\text { Towing Tank }\end{array}$ & 69 & 2.76 & 3.14 & 68.9 & 2.76 & 3.14 & 68.89 & 2.76 & 2.64 \\
\hline $\begin{array}{l}\text { Solent Towing } \\
\text { Tank }\end{array}$ & 30 & 1.7 & 1.4 & 29.95 & 1.70 & 1.40 & 29.50 & 1.70 & 1.46 \\
\hline
\end{tabular}

\subsection{Predicted Tank Transfer Functions with First Order Corrections}

The simulated transfer functions between reference and receiver hydrophones, of the three towing tanks described in Table 4, can be seen in Figure 13. For all of the Tanks, the three scales of periodicity are observable, which again can be approximated using Equations 3.3 to 3.5. The medium and small period oscillations in the frequency response function fluctuate around the slow frequency variation due to Lloyd's mirror interference at high frequencies. At low frequencies, there is a bias above the dominant Lloyd's mirror interference level, due to wave curvature effects at small $k z_{t}$.

Modal peaks below the cut-on frequency approximated by using Equation 1.1 are visible for all tanks in Figure 13. This suggest that the lossy boundary conditions of the water-solid interface allow modes to propagate below the cut-on frequency predicted using a hard wall boundary condition. As the range $r$, is small for each wall of each tank, the pressure at the reference and receiver hydrophone, will not be influenced by the lateral wave field. 

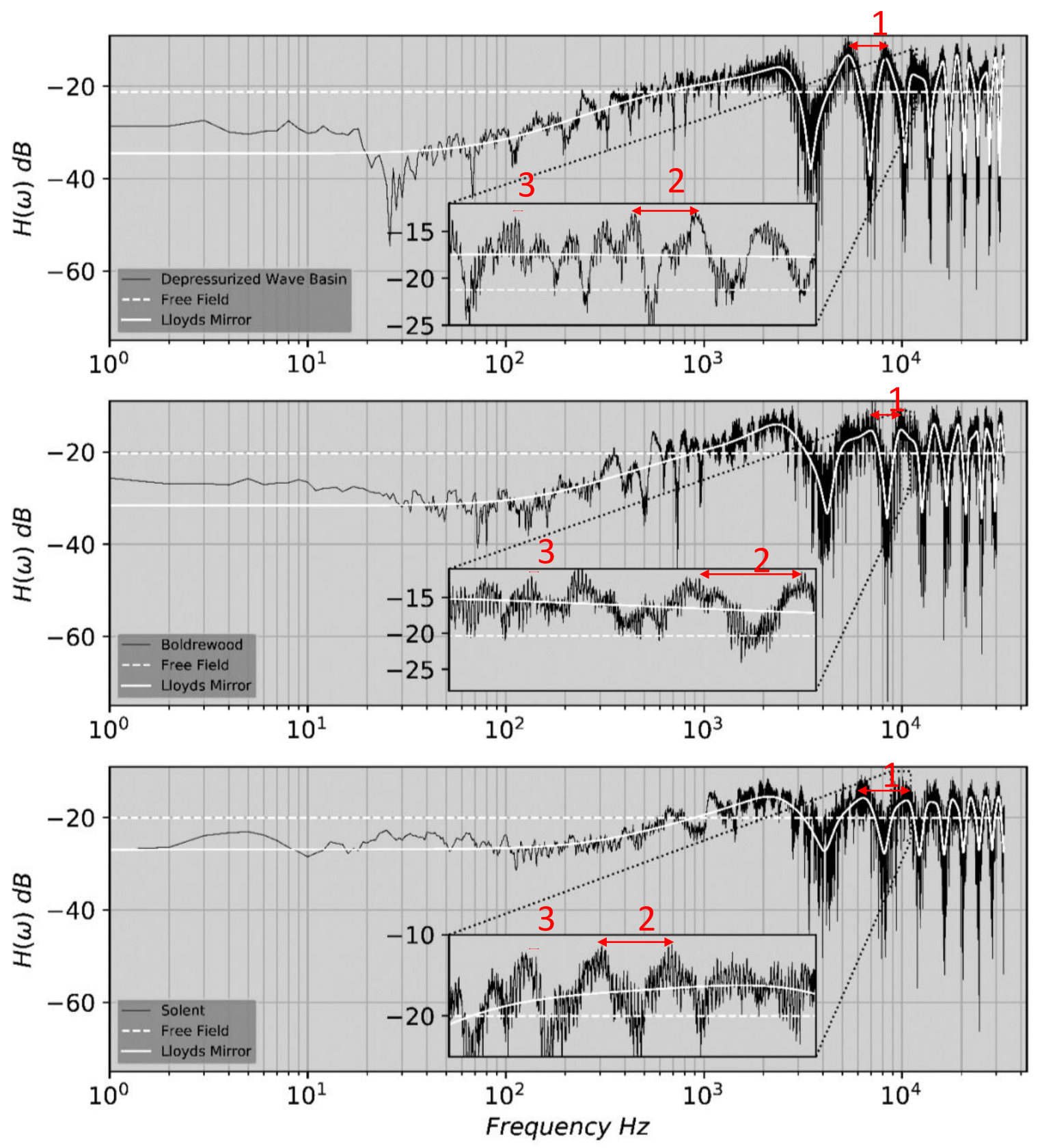

Figure 13 Transfer functions between reference and receiver hydrophones for three towing tanks. Hydrophone locations are as given in Table 4. Overlaid are the analytical free field and Lloyd's mirror transfer functions in white.

\section{Conclusions}

This paper describes an image model for the prediction of the acoustic field in a long towing tank. Interactions with the solid walls of the tank are included by the use of plane wave reflection coefficients, taking into account the excitation of compressional and shear waves in the tank walls. It is shown that an 
incident plane and spherical wave can excite compressional and shear surface waves, which propagate along the fluid-solid interface of a towing tank's concrete walls. These surface waves then excite lateral waves in the fluid, which radiate into the fluid at the critical angles of the interface.

The plane wave description is shown to be inaccurate for the $1^{\text {st }}$ order images, where wave curvature means that the incident wave cannot be approximated as a plane wave, and interference from the excited lateral wave field must be considered. Significant differences $(>10 \mathrm{~dB}$ ) between the two formulations are found when image sources are within 240 wavelengths of the receiver, with greatest difference when the receiver is placed inside the lateral wave field of each interface. A derived frequency dependent effectivespherical-wave reflection coefficient is therefore used in the adapted Image Source Model, for the first order images which represent a single reflection from each of the 5 reflecting boundaries.

Predictions from the image model are compared against published acoustic measurements and excellent qualitative agreement is obtained. Acoustic predictions are also presented for two smaller towing tanks in Southampton.

The frequency variation of the transfer function between a reference and receiver hydrophone, is shown to exhibit three periodicities. The slowest is due to the Lloyd's mirror effect arising from interference between the direct acoustic field and reflections from the free surface. The next fastest variability arises from the cut on of transverse modes between the sides of the tank and between the tank floor and free surface. The fastest oscillations in the frequency spectrum is due to interference between axial standing waves that occur between the two end walls of the tank.

This understanding and characterisation of the acoustic field of a towing tank should lead to methods for removing the tank response to recover the free field behaviour of the source, such as its sound power radiation and free field directivity. The developed image source model can be used to find the best position of a hydrophone, such that the pressure measurements are not influenced by the lateral wave field, with further reverberation removed by averaging out the three scales of frequency variability. This computational method allows high resolution pressure distributions in the towing tank to be calculated, leading to a method of calculating acoustic particle velocity using finite differences in pressure. With particle velocity calculated, acoustic intensity of the source can be predicted, and used to show the directivity of the acoustic energy loss, over an enclosing volume around the source. This knowledge can lead to a free field prediction of acoustic power.

\section{Acknowledgments}

This work is supported by Dstl through an EPSRC industrial case studentship, voucher code 16000010. Many thanks to Frans Hendrik Lafeber from MARIN for sharing the data acquired in the SONIC project, which was funded by the 7th Framework Programme (FP7) of the European Com-mission under Grant Agreement no. 314394. The authors acknowledge the use of the IRIDIS High Performance Computing Facility, and associated support services at the University of Southampton, in the completion of this work.

\section{References}

[1] J. Van Der Kooij and A. De Bruijn, "ACOUSTIC MEASUREMENTS IN THE NSMB DEPRESSURIZED TOWING TANK,” Int. Shipbuild. Prog., vol. 31, no. 353, 1984. 
[2] F. H. Lafeber, J. Bosschers, C. de Jong, and F. Graafland, "Acoustic reverberation measurements in the Depressurized Wave Basin," Adv. Model Meas. Technol. Marit. Ind., 2015.

[3] L. M. Brekhovskikh and Y. P. Lysanov, Fundamentals of Ocean Acoustics. 2003.

[4] J. B. Allen and D. A. Berkley, "Image method for efficiently simulating small-room acoustics," $J$. Acoust. Soc. Am., vol. 65, no. 4, pp. 943-950, 1979.

[5] F. H. Fisher and V. P. Simmons, "Sound absorption in sea water," Acoust. Ambient Noise Ocean Spectra Sources J. Acoust. Soc. Am., vol. 62, p. 1936, 1977.

[6] E. A. Lehmann and A. M. Johansson, "Diffuse reverberation model for efficient image-source simulation of room impulse responses," IEEE Trans. Audio, Speech Lang. Process., vol. 18, no. 6, pp. 1429-1439, 2010.

[7] E. De Sena, N. Antonello, M. Moonen, and T. Van Waterschoot, "On the modeling of rectangular geometries in room acoustic simulations," IEEE/ACM Trans. Audio Speech Lang. Process., vol. 23, no. 4, pp. 774-786, 2015.

[8] L. Brekhovskikh, Waves in Layered Media 2e, 2nd ed., vol. 16. 2012.

[9] E. K. Westwood, "Complex Ray Methods For Acoustic Interaction at A Fluid-Fluid Interface," $J$. Acoust. Soc. Am., vol. 85, no. 5, pp. 1872-1884, May 1989.

[10] P. Virtanen et al., "SciPy 1.0--Fundamental Algorithms for Scientific Computing in Python," Jul. 2019.

[11] R. Stoneley, “The Effect of the Ocean on Rayleigh Waves.," Geophys. J. Int., vol. 1, pp. 349-356, Jun. 1926.

[12] P. D. Welch, "The Use of Fast Fourier Transform for the Estimation of Power Spectra: A Method Based on Time Averaging Over Short, Modified Periodograms," IEEE Trans. Audio Electroacoust., vol. 15, no. 2, pp. 70-73, 1967. 